\title{
Trends in Stage-Specific Incidence Rates for Urothelial Carcinoma of the Bladder in the United States: 1988 to 2006
}

\author{
Matthew E. Nielsen, MD, MS ${ }^{1,2,3}$, Angela B. Smith, MD ${ }^{1,2}$, Anne-Marie Meyer, PhD ${ }^{1,3}$, Tzy- \\ Mey Kuo, PhD ${ }^{1}$, Seth Tyree, MS ${ }^{1}$, William Y. Kim, MD ${ }^{1,4}$, Matthew I. Milowsky, MD ${ }^{1,4}$, Raj S. \\ Pruthi, $\mathbf{M D}^{1,2}$, and Robert C. Millikan, $\mathbf{P h D}^{1,3, \dagger}$ \\ ${ }^{1}$ Lineberger Comprehensive Cancer Center, University of North Carolina, Chapel Hill, North \\ Carolina \\ ${ }^{2}$ Department of Urology, University of North Carolina School of Medicine, Chapel Hill, North \\ Carolina \\ ${ }^{3}$ Department of Epidemiology, University of North Carolina Gillings School of Global Public \\ Health, Chapel Hill, North Carolina \\ ${ }^{4}$ Division of Hematology/Oncology, University of North Carolina School of Medicine, Chapel Hill, \\ North Carolina.
}

\begin{abstract}
BACKGROUND: Bladder cancer is notable for a striking heterogeneity of disease-specific risks. Among the approximately $75 \%$ of incident cases found to be superficial to the muscularis propria at the time of presentation (non-muscle-invasive bladder cancer), the risk of progression to the lethal phenotype of muscle-invasive disease is strongly associated with stage and grade of disease. Given the suggestion of an increasing percentage of low-risk cases in hospital-based registry data in recent years, the authors hypothesized that population-based data may reveal changes in the stage distribution of early-stage cases.
\end{abstract}

METHODS: Surveillance, Epidemiology, and End Results (SEER) data were used to examine trends for the stage-specific incidence of bladder cancer between 1988 and 2006, adjusted for age, race, and sex, using Joinpoint and nonparametric tests.

RESULTS: The adjusted incidence rate of papillary noninvasive (Ta) predominantly low grade (77\%) disease was found to increase from 5.52 to 9.09 per 100,000 population $(P<.0001)$, with an average annual percentage change of +3.3 . Over the same period, concomitant, albeit smaller, decreases were observed for flat in situ (Tis) and lamina propria-invasive (T1) disease (2.57 to 1.19 and 6.65 to 4.61 per 100,000 population [both $P<.0001$ ]; average annual percent change of -5.0 and -1.6 , respectively). The trend was most dramatic among patients in the oldest age strata, suggesting a previously unappreciated cohort phenomenon.

CONCLUSIONS: The findings of the current study should motivate further epidemiological investigations of differential associations of genetic and environmental factors with different bladder cancer phenotypes as well as further scrutiny of clinical practice guideline

(C) 2013 American Cancer Society

Corresponding author: Matthew E. Nielsen, MD, MS, Department of Urology, University of North Carolina School of Medicine, 2107 Physicians Office Building, Campus Box 7235, Chapel Hill, NC 27599; Fax: (919) 966-0098; mnielsen@med.unc.edu.

Deceased

CONFLICT OF INTEREST DISCLOSURES

The authors made no disclosures. 
recommendations for the growing subgroup of predominantly older patients with lower-risk disease.

\section{Keywords}

bladder cancer; incidence; epidemiology; carcinogenesis; stage migration

\section{INTRODUCTION}

With a median age at diagnosis of 74 years, the burden of bladder cancer in the United States is increasing, from approximately 50,000 new cases diagnosed per year between 1990 and 2000 to $>70,000$ cases diagnosed since $2010 .{ }^{1}$ Many patients live with bladder cancer as a chronic disease, and a recent estimate of prevalent cases was $>535,000 .{ }^{1}$ Given the mandate for relatively intensive, often lifelong survivorship care ${ }^{2}$ and the comparatively indolent natural history of the disease in many cases, bladder cancer has been associated with the highest per-patient costs, from diagnosis to death, of all cancer sites. ${ }^{3}$ Incidence rates are dramatically higher in patients aged $>65$ years and peak at 179 cases per 100,000 population in patients aged 80 to 84 years, suggesting that the burden of this common cancer will continue to substantially increase as the population ages. ${ }^{1}$

As with most solid cancers, the stage and grade of the tumor at the time of presentation define the clinical significance of a bladder cancer diagnosis, and cases are first riskstratified by the presence or absence of invasion into the muscularis propria of the bladder wall. Muscle-invasive cases (classified as $2 \mathrm{~T} 2$ ) represent the lethal phenotype, with a 5-year survival rate of approximately $50 \%$, even in the face of aggressive management. ${ }^{4}$

Approximately 1 in 4 incident cases is muscle-invasive at the time of presentation. The remainder of incident, non-muscle-invasive bladder cancer (NMIBC) is characterized by uniformly high recurrence rates of NMIBC but starkly variable risks of progression to muscle-invasive disease. ${ }^{5}$ A recent hospital-based registry study suggested the possibility of an increased incidence of low-risk cases in recent years ${ }^{6}$; in this context, we hypothesized that similar dynamic changes may be underway at the population level. Given the growing burden of bladder cancer, the heterogeneous risk of different subphenotypes, and the changing age structure of the population, we sought to characterize trends in stage-specific incidence rates in the United States over the past 20 years.

\section{MATERIALS AND METHODS}

\section{Study Population}

We obtained cancer incidence data from the US National Cancer Institute (NCI)'s Surveillance, Epidemiology, and End Results (SEER) Program using the November 2011 release. ${ }^{7}$ The case and SEER population data from all 18 registries were both downloaded from the SEER Web site. To calculate incidence rates adjusted for age, race, and sex, we also downloaded Census 2000 data as the "standard population" from the Web site. ${ }^{8}$ The University of North Carolina Institutional Review Board exempted this study from review.

We subset the data to include cases of primary urinary bladder cancer that were diagnosed between 1988 and 2006 using the SEER site recode code 29010, which maps to International Statistical Classification of Diseases for Oncology (3rd edition) (ICD-O-3) topography codes 67.0 to 67.9. The analysis was restricted to cases with transitional cell/ urothelial histology, the most common type of bladder cancer, ${ }^{4}$ using ICD-O-3 codes 8120 , $8121,8122,8082,8020,8130$, and $8131 .{ }^{9}$ The study period was chosen to begin in 1988 due to the promulgation of a new 10-digit extent of disease (EOD) scheme in SEER that year, ${ }^{10}$ and terminated in 2006 due to the adoption of a new "multiple primaries" rule in 2007, 
which instructed registrars to code invasive tumors occurring $\geq 60$ days after an index noninvasive/in situ tumor as a new incident primary tumor. ${ }^{11}$ Due to changes in the reporting of tumor stage over time, a crosswalk was developed using the codes from the SEER EOD ${ }^{10,12}$ and Collaborative Staging $(C S)^{13}$ variables (Table 1). A total of 170,760 incident cases were identified. Among these, we excluded 72 cases for which the TNM T classification was not of study interest (ie, T0 [8 cases] and stage IV [64 cases]), and focused on the T classifications of Ta, Tis, $\mathrm{T} 1$, or $\geq \mathrm{T} 2$. Cases that were staged as Tx (1017 cases) and unstaged cases (3960 cases) were combined and included as a separate category for analytic purposes. Grading was defined as low (well-differentiated and moderately differentiated), high (poorly differentiated and un-differentiated), and other/unknown. Several sensitivity analyses were performed to gauge effects in changes of coding over time. To examine arbitrary shifts in incidence rates over time, we performed a sensitivity analysis reclas-sifying low-grade Tis cases to Ta because Tis cases are, by clinical definition, high grade only. ${ }^{4}$ In addition, we performed a sensitivity analysis reclassifying Tx/unstaged cases as Ta to confirm that the magnitude of changes in this group over the study period did not account for the observed changes in the specified stages.

\section{Statistical Analysis}

From the SEER data, we summed the number of cases for each TNM T classification and the underlying SEER population for each of the strata defined by age, race, and sex within each year across the study period. Specifically, age was grouped into 7 categories $(<34$ years, 35-44 years, $45-54$ years, 55-64 years, $65-74$ years, $75-84$ years, and $\geq 85$ years); race was coded as white, black, or other; and sex was coded as male or female. We also summed the number of people from the standard population for each of the corresponding strata described above to weight stratum-specific rates to calculate adjusted rates. The data set contained covariates describing T classification, case count, SEER population count, standard population count, year, and strata and were input into the Joinpoint Regression Program (version 4.0.1; NCI, Bethesda, Md) to examine trends of stage-specific incidence rates adjusted for age, race, and sex. Age strata-specific incidence rates adjusted for race and sex were also analyzed. Joinpoint regression is a linear modeling approach that fits incidence rate data (the dependent variable) for multiple years (the key independent variable) and allows adjustment for factors such as age, race, and sex while standardizing the incidence rates with the standard population. The program uses a sequence of permutation tests to select a best-fit model with the smallest number of "joinpoints." 14 The program uses the following steps to estimate model parameters and number of joinpoints: 1) a weighted least-squares regression approach is used to find a best-fit model for each possible number of joinpoints; and 2) permutation tests ${ }^{14}$ are used to determine the maximum number of joinpoints by testing the hypothesis that adding more joinpoints does not significantly improve model fit. To deal with small incidence rates, we selected a loglinear model in the Joinpoint analysis.

Although Joinpoint regression allows for a trend analysis, it focuses on within-stratum relative (percentage) changes, and does not have the flexibility to statistically test the absolute difference between 2 incidence estimates within the same stage at 2 specified time points or between 2 different stages at the same time point. To address this limitation, we also computed standard errors ${ }^{15}$ (SEER*-Stat, NCI) for the adjusted rates for each T classification over time. We then compared the differences between 2 rates using a nonparametric method proposed by Carriere and Roos ${ }^{16}$ because this approach does not require any assumptions that data originate from a known distribution. Specifically, this method computes a Hotelling's T-squared $\left(\mathrm{T}^{2}\right)$ statistic, which follows a chi-square distribution with a large sample. In a test comparing 2 rates, it is operationalized as a squared $t$ statistic for the 2-sample Student $t$ test. All analyses except the Joinpoint 
regression models were performed using SAS statistical software (SAS Institute Inc, Cary, $\mathrm{NC})$.

\section{RESULTS}

The final cohort included 165,711 incident cases of staged primary urothelial carcinoma of the bladder (hereafter referred to as bladder cancer) diagnosed between in 1988 and 2006 in the 18 SEER registries. Among these, $79 \%$ of the cases were diagnosed at early stage (45\% with Ta disease, $24 \%$ with $\mathrm{T} 1$ disease, and $10 \%$ with Tis disease) whereas $21 \%$ were diagnosed at late stage ( $\geq T 2)$ (Table 2).

The incidence rate adjusted for age, race, and sex (hereafter referred to as the adjusted incidence rate) per 100,000 for bladder cancer of all stages was relatively stable (from 18.6 in 1988 to 18.8 in 2006) (Fig. 1). Similarly stable rates were observed when stratified by early $(<\mathrm{T} 2)$ versus late ( $\geq \mathrm{T} 2)$ stage (Fig. 1). However, the stage-stratified adjusted rates within early-stage disease demonstrated a dramatic rise in the adjusted incidence rate of noninvasive (Ta) disease, of which $77 \%$ were low grade (from 5.52 to 9.09) with concomitant, albeit lesser in magnitude, decreases in the incidence rates for Tis (from 2.57 to 1.19 ) and T1 (6.65 to 4.61) disease (all $P<.0001$ ) (Table 3) (Fig. 2). ${ }^{14}$ Unstaged/stage Tx cases represented a small minority ( $2.9 \%$ of the total cohort) and the adjusted incidence rates in this group decreased over the study period (from 0.73 to $0.37 ; P<.0001$ ) (Fig. 2).

The trends from Joinpoint regression analysis (Table 3) (Fig. 2) demonstrated that adjusted incidence rates of Ta continuously increased between 1988 and 2006. ${ }^{14}$ The largest increase occurred before 1991 (average annual percentage change [AAPC] of $+11.2 \%$ ), and slowed down after 1991 (AAPC of $+1.8 \%$ ). In contrast, Tis decreased slowly before 2003 (AAPC of $-3.0 \%$ ) and then accelerated from 2003 to 2006 (AAPC of $-14.4 \%$ ). The trend for T1 disease was less consistent, with an AAPC of $-4.6 \%$ from 1988 to 1997, followed by an $\mathrm{AAPC}$ of $+1.6 \%$. Finally, the trend for $\geq \mathrm{T} 2$ disease increased at a constant and slow rate of $0.1 \%$ annually, and the rate for Tx/unstaged disease decreased with an AAPC of 5.1.

The stage-specific trends within age strata, adjusted for race and sex, revealed substantially higher incidence rates for all stages of disease, as well as the greatest absolute and relative increases of Ta incidence, among the older age strata (Fig. 3). The AAPC was +1.3 for those aged 55 to 64 years and was over +3.0 for all other older age groups; this indicates that the trend increase for Ta disease is more significant for older age groups than for the younger age group. Joinpoint regression analysis for parallelism also suggested that the trends in $\mathrm{Ta}$ incidence between all age groups were not parallel except among the 2 oldest age groups (aged 75 years- 84 years and aged $\geq 85$ years; data not shown). Because the incidence rates were very low for individuals aged $<55$ years, data presented in the figure include only the age strata for age $\geq 55$ years.

In sensitivity analyses (Table 4) (Fig. 4A), when we reclassified Ta and Tis by moving the low-grade Tis to Ta, the magnitude of the increase in the adjusted incidence of Ta over the study period was attenuated but was still statistically significant (7.31 to 9.55 per 100,000; $P$ $<.0001) .{ }^{14}$ With this reclassification, the incidence rate of Tis was more stable over time (from 0.78 to 0.73 per 100,000). When we reclassified unstaged/Tx disease to Ta, the magnitude of the increase in the adjusted incidence of Ta was attenuated but still statistically significant (6.25 to 9.46 per 100,000; $P<.0001$ ) (Table 4) (Fig. 4 B). ${ }^{14}$ 


\section{DISCUSSION}

We observed a shift in the stage-specific, age-, race-, and gender-adjusted incidence rates of early stage bladder cancer in the United States over the past 20 years, with increasing absolute and relative percentages of newly diagnosed cases of Ta disease (noninvasive papillary), which is low grade in nearly $80 \%$ of cases. The proportional changes observed in SEER data are consistent with results from a prior large hospital-based registry study conducted between 1993 and $2007 . .^{6}$ To the best of our knowledge, the current study is the first to document this phenomenon in population-based data, affording the ability to measure incidence rates, and demonstrates a statistically significant stage-specific increase in the rate of noninvasive disease in analyses adjusted for age, race, and sex during this period (Fig. 2) (Table 3). The most dramatic relative and absolute increases in Ta incidence occurred among the oldest age strata, in which the overall burden of disease is also the highest (Fig. 3 ). The high, and rising, incidence rates in the elderly suggest that the burden of disease will dramatically increase with the changing age structure of the population in coming decades. ${ }^{17}$ To the best of our knowledge, in contrast to other disease sites in which stage migration has been documented, bladder cancer has rarely been detected incidentally at autopsy and there has been no systematic screening program or fundamental change in the technology leading to the diagnosis of bladder cancer (cystoscopy and transurethral resection of bladder tumor) over the past 50 years. ${ }^{4}$ Therefore, these findings raise fundamental questions with regard to bladder cancer epidemiology.

Because disease incidence is sensitive to changes in disease reporting, we examined changes in grade and stage over the study period, particularly focusing on the classification of carcinoma in situ, which is, by clinical definition, high grade only. ${ }^{4}$ When we reclassified cases registered as low-grade Tis to Ta, the magnitude of the increase in Ta was slightly attenuated, although still statistically significant (Fig. 4A) (Table 4). Further-more, although the adjusted incidence rate of unstaged/Tx cases decreased over the study period, these cases represented a very small percentage of the overall cohort, and a sensitivity analysis reclassifying these cases as Ta did not change the overall findings (Fig. 4B), suggesting the observed trends are not an artifact of more complete staging performed in later years of the study. These findings underscore the proportionally large and increasing percentage of cases of relatively lower risk and suggest that the shifts in incidence observed in the current study are not attributable to changes in disease definition but rather epidemiologic changes in the population.

There is substantial variability in the risk of progression to the lethal phenotype of muscleinvasive ( $\geq \mathrm{T} 2$ ) disease among the approximately $75 \%$ of patients initially presenting with NMIBC. Patients with low-grade Ta tumors tend to have recurrent low-grade Ta tumors, at rates of $30 \%$ to $70 \%$ by 5 years. ${ }^{5,18,19}$ In contrast, the 5 -year risk of progression to $\geq \mathrm{T} 2$ disease in these patients is $\leq 5 \%$, with subgroups in the largest series showing rates of < $1 \% .^{5}$ In fact, the rates of recurrent, low-risk, noninvasive disease; progression to high-grade NMIBC ${ }^{19}$; and invasive ( $\geq \mathrm{T} 2$ ) cancer for patients with low-grade Ta bladder cancer are similar to those reported in a pooled analysis of colorectal adenoma studies for recurrent adenoma, progression to dysplastic polyp, and invasive ( $\geq \mathrm{T} 2$ ) cancer, respectively. ${ }^{20}$ This is in stark contrast to $\geq \mathrm{T} 2$ progression rates of $30 \%$ to $70 \%$ in series of high-grade (T1 and/or Tis disease) NMIBC. 5,18 Given these dramatic differences in risk, the findings of the current study in contemporary epidemiologic data suggesting a disproportionately increasing fraction of elderly patients with low-risk pathology raise questions regarding not only the etiology of this phenomenon but also the implications of these trends on cancer control policy, given the burdens of survivorship care on patients and the health care system. ${ }^{3}$ 
The observed clinical heterogeneity in this context motivated a body of molecular studies implicating parallel, infrequently overlapping, biological pathways in the carcinogenesis of the different phenotypes. As with many solid tumors, genomic instability and alterations in cell cycle regulatory pathways are common among the poorer-risk sub-group, ${ }^{21,22}$ although these are rare among patients with low-grade noninvasive disease. ${ }^{22}$ Activating mutations of fibro-blast growth factor receptor 3 (FGFR3), similar to those found in congenital skeletal dysplasias, have emerged as a prevalent (60\%-95\%) anomaly, ${ }^{22-24}$ rarely seen in other tumor types. ${ }^{25}$ It is interesting to note that these mutations appear to be specific for favorable-risk disease, ${ }^{22,26}$ reported to be mutually exclusive with p53 mutations detected in 94\% of cases in 1 study. ${ }^{22}$ Building on these findings, several different FGFR3-based "molecular grading" schemes have been investigated, which appear to offer superior reproducibility and enhanced prognostic classification compared with standard clinicopathological parameters. ${ }^{27}$

Given the relatively biphasic biology associated with different clinical phenotypes, our observation of a specific increase in the incidence of lower-risk disease begs the question of how differences in carcinogenesis might explain the observed trends. The classic risk factors for bladder cancer are age, male sex, and environmental exposures, in particular cigarette smoke ${ }^{28}$ and occupational exposures to aromatic amines. ${ }^{29}$ Data from population-based case-control studies in the $1960 \mathrm{~s}^{30}$ and $1970 \mathrm{~s}^{31}$ suggested that early-stage and invasive bladder cancer share most suspected risk factors. However, more recently, it was suggested that smoking may be more strongly associated with the risk of invasive cancer than lowgrade superficial disease. ${ }^{32}$ It is interesting to note that smoking has also been specifically associated with an increased risk of TP53 mutations but not activating mutations in FGFR $3{ }^{33}$ Several single-nucleotide polymorphisms have been associated with the specific subphenotypes of low-grade and high-grade NMIBC and muscle-invasive disease. ${ }^{34}$ Further research in more contemporary population-based cohorts is needed to assess the interaction between different genotypes and exposure histories for the different clinical phenotypes.

Given our finding of a dramatically increasing incidence of noninvasive disease among the oldest age strata, suggestive of a cohort effect (Fig. 3), age-period-cohort modeling represents an attractive approach with which to explore the hypotheses generated in this study. ${ }^{35}$ This approach has been used in this context to evaluate the contribution of temporal changes in smoking as a driver of trends ${ }^{36}$; updating this work in contemporary data may be particularly relevant given evidence of a recent increasing trend in smoking-related bladder cancer risk, which suggests a potential period effect. ${ }^{37}$

The standard clinical management of patients with NMIBC includes transurethral resection of the tumor followed by periodic surveillance cystoscopy; however, evidence informing specific surveillance regimens is sparse, and practice guidelines are largely based on the consensus of expert opinion. ${ }^{18} \mathrm{~A}$ consideration of the discordance between the recommendations of relevant guidelines for the surveillance of patients newly diagnosed with low-grade noninvasive bladder cancer highlights the uncertainty of management decision-making. The European guidelines explicitly stratify patients based on risk and recommend a specific, less aggressive surveillance schedule for patients with low-risk disease. ${ }^{38}$ The American guidelines state that the most common approach includes patient assessment performed every 3 months within the first 2 years after the initial diagnosis followed by assessment every 6 months for the next 2 to 3 years and then annually thereafter. ${ }^{2}$ Although the American guidelines do state that "a risk-adapted approach should be considered," 2 no specific alternative schedule is outlined, leaving the relatively intensive regimen as the default for all patients. Although this approach is certainly justifiable among the subgroup of patients with high-risk (T1 and Tis) NMIBC, for which the surveillance guidelines are concordant, ${ }^{2,38}$ our findings of high and increasing incidence rates for low- 
risk noninvasive papillary disease, predominantly among the oldest age strata, suggest that a critical reevaluation of the essentially " 1 size fits all" approach of the US clinical practice guidelines may be warranted.

The strengths of the current study include the population-based sample, which facilitates the calculation of adjusted incidence rates and supports the generalizability of these findings. In addition, the restriction to those cases with urothelial histology supports our speculation regarding risk group-specific carcinogenesis, as discussed above, as a potential explanatory hypothesis. In sensitivity analyses, we found similar results with all bladder cancer cases, with the exception of a parallel but higher incidence trend for $\geq \mathrm{T} 2$ disease when including nonurothelial subtypes, in which the percentage of $\geq \mathrm{T} 2$ disease was $58 \%$ versus $21 \%$ in urothelial-only subtypes (data not shown). These observational hypothesis-generating data have limitations, including the possibility of misclassification or unmeasured changes in stage reporting practices over time. We evaluated this in our sensitivity analyses and the main result was still statistically significant. Nonetheless, the possibility of misclassification remains, and future work with confirmatory centralized pathology review is needed. We recognize that the stage crosswalk using SEER EOD/CS codes may misclassify some cases; however, the consistency of our observed proportional changes over time with those reported in another large independent data set using American Joint Committee on Cancer TNM stage data ${ }^{6}$ are reassuring. Although to the best of our knowledge there has been no systematic screening program or fundamental technological changes in the diagnosis of bladder cancer over the study period, ${ }^{4}$ it is also possible that some as-yet unappreciated secular changes in the diagnostic approach to hematuria in elderly patients may be underway, given demographic changes and our observation of concomitant, albeit lower in magnitude, decreases in the incidence of $\mathrm{T} 1$ and Tis cases. We adjusted for age, race, and sex, which are well-recognized risk factors for overall diagnosis, stage at presentation, and survival. ${ }^{6,39}$ Hispanic ethnicity has also been associated with a lower risk of bladder cancer, although with similar stage distribution at diagnosis and survival, compared with nonHispanic Caucasians. ${ }^{39}$ SEER data regarding this variable are incomplete for the period of interest, but we found that adding an adjustment for this variable among cases for which there was available information did not significantly alter the findings ( $4.5 \%$ of cases had Hispanic ethnicity; data not shown).

\title{
Conclusions
}

In this descriptive, hypothesis-generating study, we confirmed, using population-based data, the suggestion from a prior hospital-based registry study of increases in the relative and absolute incidence of noninvasive papillary ( $\mathrm{Ta}$ ) urothelial carcinoma of the bladder in recent years. These findings support the need for further epidemiological study of potential mechanisms, as well as scrutiny of the recommendations of clinical practice guidelines for this large and growing population of predominantly elderly patients with lower-risk disease.

\section{Acknowledgments}

\author{
The authors would like to acknowledge the anonymous reviewers, whose constructive feedback resulted in \\ substantial improvements to the study. \\ FUNDING SUPPORT \\ Supported by the University Cancer Research Fund at the University of North Carolina Lineberger Comprehensive \\ Cancer Center (to Drs. Nielsen, Smith, Meyer, Kuo, Tyree, Milowsky, and Pruthi) and the University of North \\ Carolina KL2 Scholars Program (National Institutes of Health grant 1KL2RR025746 to Drs. Nielsen and Smith).
}




\section{REFERENCES}

1. Jemal A, Siegel R, Xu J, Ward E. Cancer statistics. CA Cancer J Clin. Sep-Oct;2010 60(5):277-300. [PubMed: 20610543]

2. Hall, MC.; Chang, SS.; Dalbagni, G., et al. AUA Bladder Cancer Clinical Guidelines Panel Summary Report on the Management of Nonmuscle Invasive Bladder Cancer. http:// www.auanet.org/content/guidelines-and-quality-care/clinical-guidelines/main-reports/bladcan07/ chapter1.pdf. Accessed October 27, 2010

3. Riley GF, Potosky AL, Lubitz JD, Kessler LG. Medicare payments from diagnosis to death for elderly cancer patients by stage at diagnosis. Med Care. Aug; 1995 33(8):828-841. [PubMed: 7637404]

4. Messing, EM. Urothelial Tumors of the Bladder. In: Wein, A., editor. Campbell-Walsh Urology. 9th. Vol. 3. Elsevier / Saunders; Philadelphia: 2007. p. 2406-2445.

5. Sylvester RJ, van der Meijden AP, Oosterlinck W, et al. Predicting recurrence and progression in individual patients with stage Ta T1 bladder cancer using EORTC risk tables: a combined analysis of 2596 patients from seven EORTC trials. Eur Urol. Mar; 2006 49(3):466-465. discussion 475-467. [PubMed: 16442208]

6. David KA, Mallin K, Milowsky MI, Ritchey J, Carroll PR, Nanus DM. Surveillance of urothelial carcinoma: stage and grade migration, 1993-2005 and survival trends, 1993-2000. Cancer. Apr 1; 2009 115(7):1435-1447. [PubMed: 19215030]

7. Surveillance, Epidemiology, and End Results (SEER) Program. www.seer.cancer.govResearch Data (1973-2009), National Cancer Institute, DCCPS, Surveillance Research Program, Surveillance Systems Branch, released April 2012, based on the November 2011 submission. April 2011 (updated 10/28/2011), based on the November 2010 Submission ed; 2011

8. Bureau, C. American Fact Finder. http:factfinder2.census.gov. Accessed February 1, 2013

9. SEER. ICD-O-3 SEER Site / histology validation. Dec 5. 2012 http://seer.cancer.gov/icd-o-3/ sitetype.icdo3.d20121205.pdf. Accessed March 1, 2013

10. Shambaugh, EM.; Gloeckler, Ries L.; Young, JL.; Kruse, MA. SEER Extent of Disease-1988 Codes and Coding Instructions. p. 126http://seer.cancer.gov/manuals/historic/EOD_1988.pdf. Accessed April 4, 2012

11. Johnson, CH.; Adamo, M. SEER Program Coding and Staging Manual 2007. 2008. Appendix C, part 6, page C-814revision:http://seer.cancer.gov/manuals/2007/SPCSM_2007_maindoc.pdf. Accessed April 4, 2012

12. Fritz, A.; Ries, L. SEER Extent of Disease-1988 Codes and Coding Instructions. 3rdhttp:// seer.cancer.gov/manuals/EOD10Dig.pub.pdf. Accessed April 4, 2012

13. Johnson, CH. SEER Program Coding and Staging Manual 2004. revision 1, Appendix C, part 3: Site-specific coding modules, page C-601. http://seer.cancer.gov/manuals/2004Revision\%201/ SPM_AppendixC_Part3_r1.pdf. Accessed April 4, 2012

14. Kim HJ, Fay MP, Feuer EJ, Midthune DN. Permutation tests for joinpoint regression with applications to cancer rates. Stat Med. Feb 15; 2000 19(3):335-351. [PubMed: 10649300]

15. Tiwari RC, Clegg LX, Zou Z. Efficient interval estimation for age-adjusted cancer rates. Stat Methods Med Res. Dec; 2006 15(6):547-569. [PubMed: 17260923]

16. Carriere KC, Roos LL. Comparing standardized rates of events. Am J Epidemiol. Sep 1; 1994 140(5):472-482. [PubMed: 8067339]

17. U.S. Census Bureau. U.S. Interim Projections by Age, Sex, Race, and Hispanic Origin: 2000-2050. http://www.census.gov/population/www/projections/summarytables.html. Accessed October 9, 2011

18. Donat SM. Evaluation and follow-up strategies for superficial bladder cancer. Urol Clin North Am. Nov; 2003 30(4):765-776. [PubMed: 14680313]

19. Herr HW, Donat SM, Reuter VE. Management of low grade papillary bladder tumors. J Urol. Oct. 2007 178:1201-1205. 4 Pt 1. discussion 1205. [PubMed: 17698090]

20. Martinez ME, Baron JA, Lieberman DA, et al. A pooled analysis of advanced colorectal neoplasia diagnoses after colonoscopic polypectomy. Gastroenterology. Mar; 2009 136(3):832-841.

[PubMed: 19171141] 
21. Primdahl H, von der Maase H, Christensen M, Wolf H, Orntoft TF. Allelic deletions of cell growth regulators during progression of bladder cancer. Cancer Res. Dec 1; 2000 60(23):6623-6629. [PubMed: 11118045]

22. van Rhijn BW, van der Kwast TH, Vis AN, et al. FGFR3 and P53 characterize alternative genetic pathways in the pathogenesis of urothelial cell carcinoma. Cancer Res. Mar 15; 2004 64(6):19111914. [PubMed: 15026322]

23. van Rhijn BW, van Tilborg AA, Lurkin I, et al. Novel fibroblast growth factor receptor 3 (FGFR3) mutations in bladder cancer previously identified in non-lethal skeletal disorders. Eur J Hum Genet. Dec; 2002 10(12):819-824. [PubMed: 12461689]

24. Cappellen D, De Oliveira C, Ricol D, et al. Frequent activating mutations of FGFR3 in human bladder and cervix carcinomas. Nat Genet. Sep; 1999 23(1):18-20. [PubMed: 10471491]

25. Sibley K, Stern P, Knowles MA. Frequency of fibroblast growth factor receptor 3 mutations in sporadic tumours. Oncogene. Jul 19; 2001 20(32):4416-4418. [PubMed: 11466624]

26. Hernandez S, Lopez-Knowles E, Lloreta J, et al. Prospective study of FGFR3 mutations as a prognostic factor in nonmuscle invasive urothelial bladder carcinomas. J Clin Oncol. Aug 1; 2006 24(22):3664-3671. [PubMed: 16877735]

27. van Rhijn BW, Vis AN, van der Kwast TH, et al. Molecular grading of urothelial cell carcinoma with fibroblast growth factor receptor 3 and MIB-1 is superior to pathologic grade for the prediction of clinical outcome. J Clin Oncol. May 15; 2003 21(10):1912-1921. [PubMed: 12743143]

28. Cole P, Monson RR, Haning H, Friedell GH. Smoking and cancer f the lower urinary tract. N Engl J Med. Jan 21; 1971 284(3):129-134. [PubMed: 5538681]

29. Cole P, Hoover R, Friedell GH. Occupation and cancer of the lower urinary tract. Cancer. May; 1972 29(5):1250-1260. [PubMed: 5021618]

30. Hayes RB, Friedell GH, Zahm SH, Cole P. Are the known bladder cancer risk-factors associated with more advanced bladder cancer? Cancer Causes Control. Mar; 1993 4(2):157-162. [PubMed: 8481494]

31. Sturgeon SR, Hartge P, Silverman DT, et al. Associations between bladder cancer risk factors and tumor stage and grade at diagnosis. Epidemiology. Mar; 1994 5(2):218-225. [PubMed: 8172997]

32. Jiang X, Castelao JE, Yuan JM, et al. Cigarette smoking and sub-types of bladder cancer. Int J Cancer. Feb 15; 2012 130(4):896-901. [PubMed: 21412765]

33. Wallerand H, Bakkar AA, de Medina SG, et al. Mutations in TP53, but not FGFR3, in urothelial cell carcinoma of the bladder are influenced by smoking: contribution of exogenous versus endogenous carcinogens. Carcinogenesis. Jan; 2005 26(1):177-184. [PubMed: 15347601]

34. Guey LT, Garcia-Closas M, Murta-Nascimento C, et al. Genetic susceptibility to distinct bladder cancer subphenotypes. Eur Urol. Feb; 2010 57(2):283-292. [PubMed: 19692168]

35. Rosenberg PS, Anderson WF. Age-period-cohort models in cancer surveillance research: ready for prime time? Cancer Epidemiol Bio-markers Prev. Jul; 2011 20(7):1263-1268.

36. Zheng T, Holford TR, Chen Y, et al. Time trend and age-period-cohort effect on incidence of bladder cancer in Connecticut, 1935-1992. Int J Cancer. Oct 9; 1996 68(2):172-176. [PubMed: 8900423]

37. Baris D, Karagas MR, Verrill C, et al. A case-control study of smoking and bladder cancer risk: emergent patterns over time. J Natl Cancer Inst. Nov 18; 2009 101(22):1553-1561. [PubMed: 19917915]

38. Babjuk M, Oosterlinck W, Sylvester R, Kaasinen E, Bohle A, Palou-Redorta J. EAU guidelines on non-muscle-invasive urothelial carcinoma of the bladder. Eur Urol. Aug; 2008 54(2):303-314. [PubMed: 18468779]

39. Yee DS, Ishill NM, Lowrance WT, Herr HW, Elkin EB. Ethnic differences in bladder cancer survival. Urology. Sep; 2011 78(3):544-549. [PubMed: 21782222] 


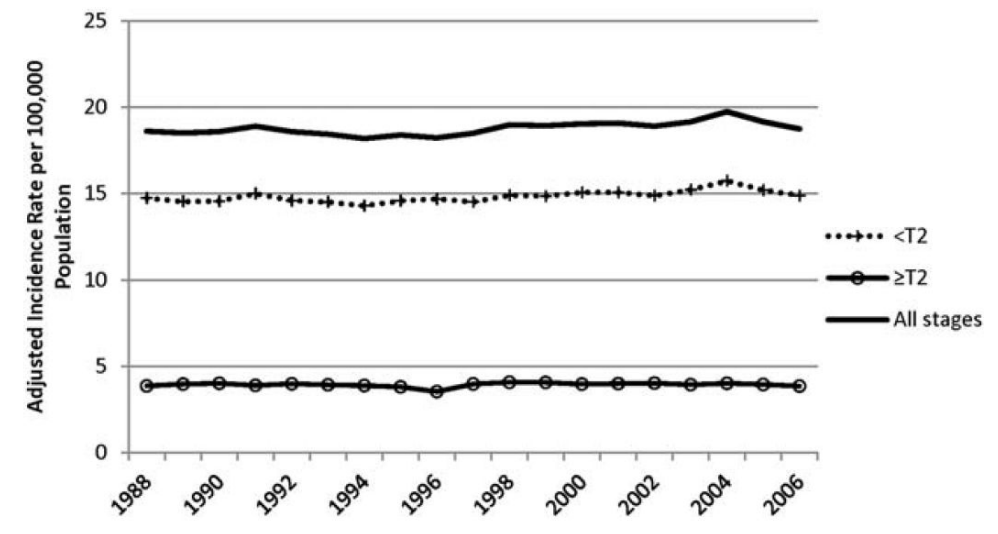

Figure 1.

Incidence rates for urothelial carcinoma of the bladder are shown adjusted for age, race, and sex for overall, early-stage, and late-stage disease, 1988 to 2006. 


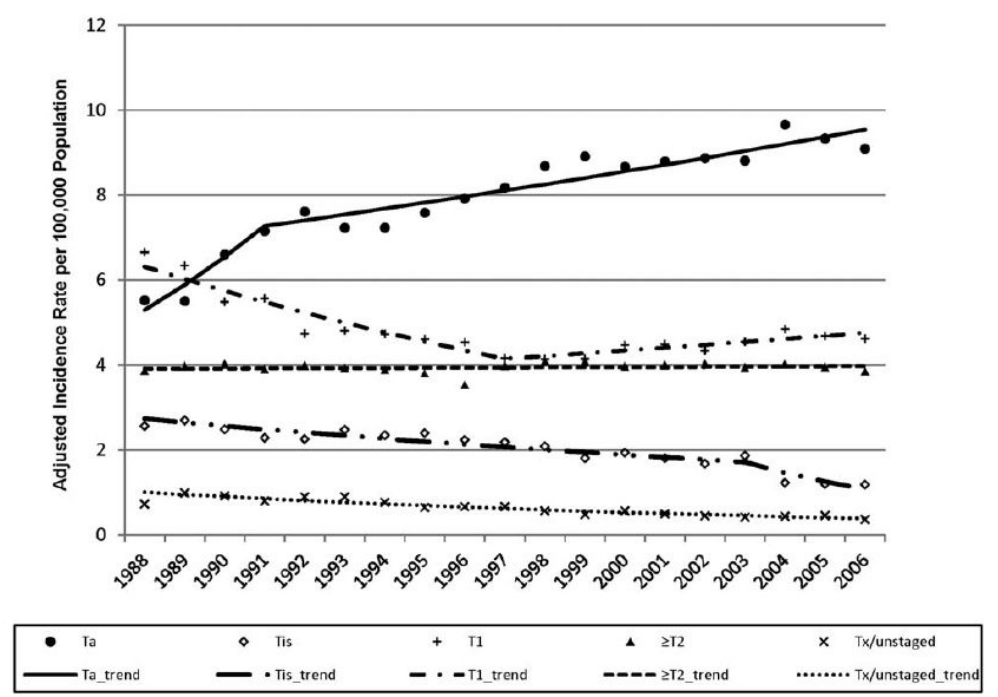

Figure 2.

Stage-specific incidence rates for urothelial carcinoma of the bladder are shown adjusted for age, race, and sex, 1988 to 2006, using Joinpoint regression analysis. 

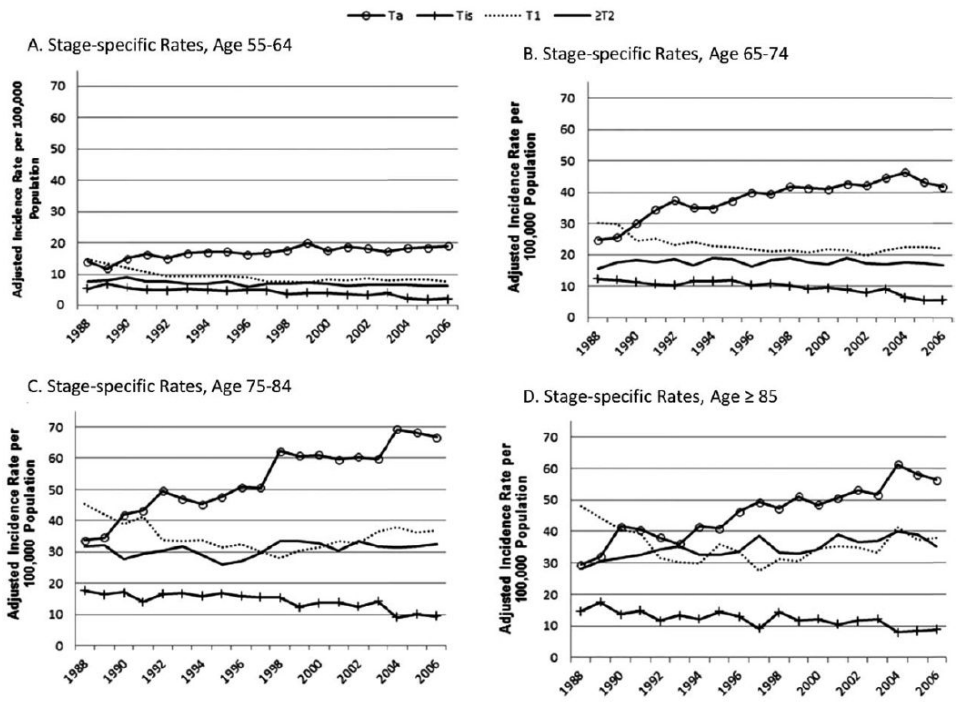

Figure 3.

Race- and sex-adjusted stage-specific incidence rates for urothelial carcinoma of the bladder are shown by age strata, 1988 to 2006. Solid line with circles indicates Ta disease; solid line with tick marks, Tis disease; dotted line, T1 disease; solid line, $\geq$ disease. 

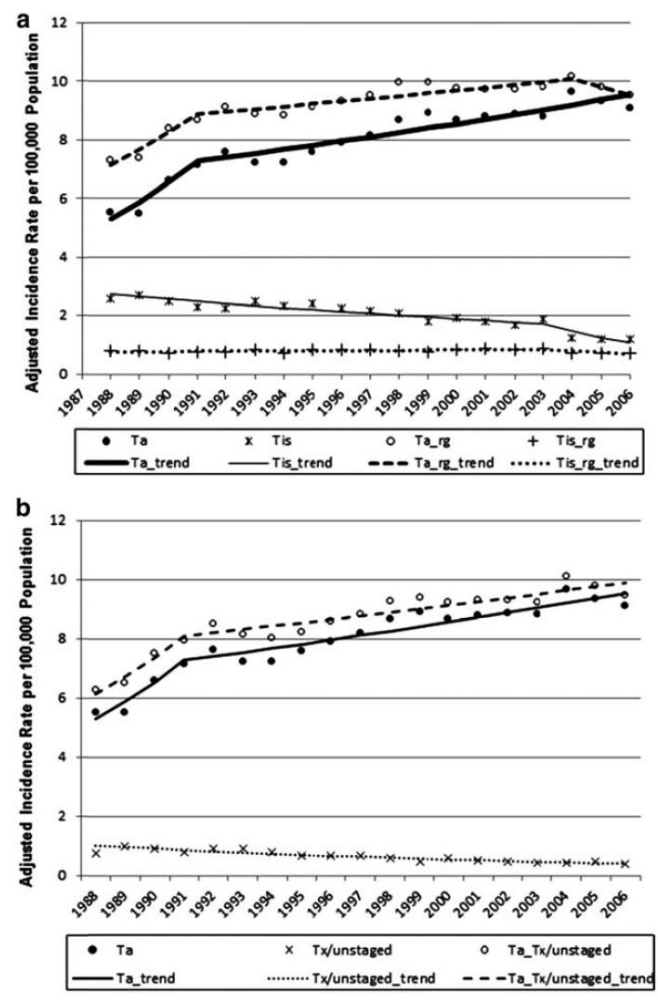

Figure 4.

Sensitivity analyses of incidence rates for urothelial carcinoma of the bladder adjusted for age, race, and sex are shown, 1988 to 2006. (A, Top) Reclassification of low-grade Tis to Ta using Joinpoint regression analysis is shown. (B, Bottom) Reclassification of Tx/unstaged to Ta disease using Joinpoint regression analysis is shown. 
TABLE 1

SEER Stage Crosswalk for Defining T Classification

\begin{tabular}{lccc}
\hline & \multicolumn{3}{c}{ Diagnosis Years } \\
\cline { 2 - 4 } T Classification & $\begin{array}{c}\mathbf{1 9 8 8 - 1 9 9 8}^{\boldsymbol{a}} \\
\text { EOD Extension, }^{\mathbf{8}} \text { 1st ed }\end{array}$ & $\begin{array}{c}\mathbf{1 9 9 9 - 2 0 0 3}^{\boldsymbol{a}} \\
\text { EOD Extension, }{ }^{\mathbf{1 0}} \text { 3rd ed }\end{array}$ & $\begin{array}{c}\mathbf{2 0 0 4 - 2 0 0 6} \\
\text { CS Extension }^{\mathbf{1 1}}\end{array}$ \\
\hline $\mathrm{Ta}$ & 05 & 01,03 & 010,030 \\
$\mathrm{Tis}$ & 00,10 & 06,10 & 060,100 \\
$\mathrm{~T} 1$ & 15,30 & 15,30 & $150,155,160,165,170,300$ \\
$\geq \mathrm{T} 2$ & $20-23,40-85$ & $20-23,40-85$ & $200-240,400-810$ \\
\hline
\end{tabular}

Abbreviations: CS, collaborative staging; EOD: extent of disease; SEER, Surveillance, Epidemiology, and End Results.

${ }^{a}$ Because the 3rd edition of the National Cancer Institute staging manual was released on January 1, 1998 and some registries had delays in adoption, we allowed for cases to be coded under the 1st or 3rd edition. 


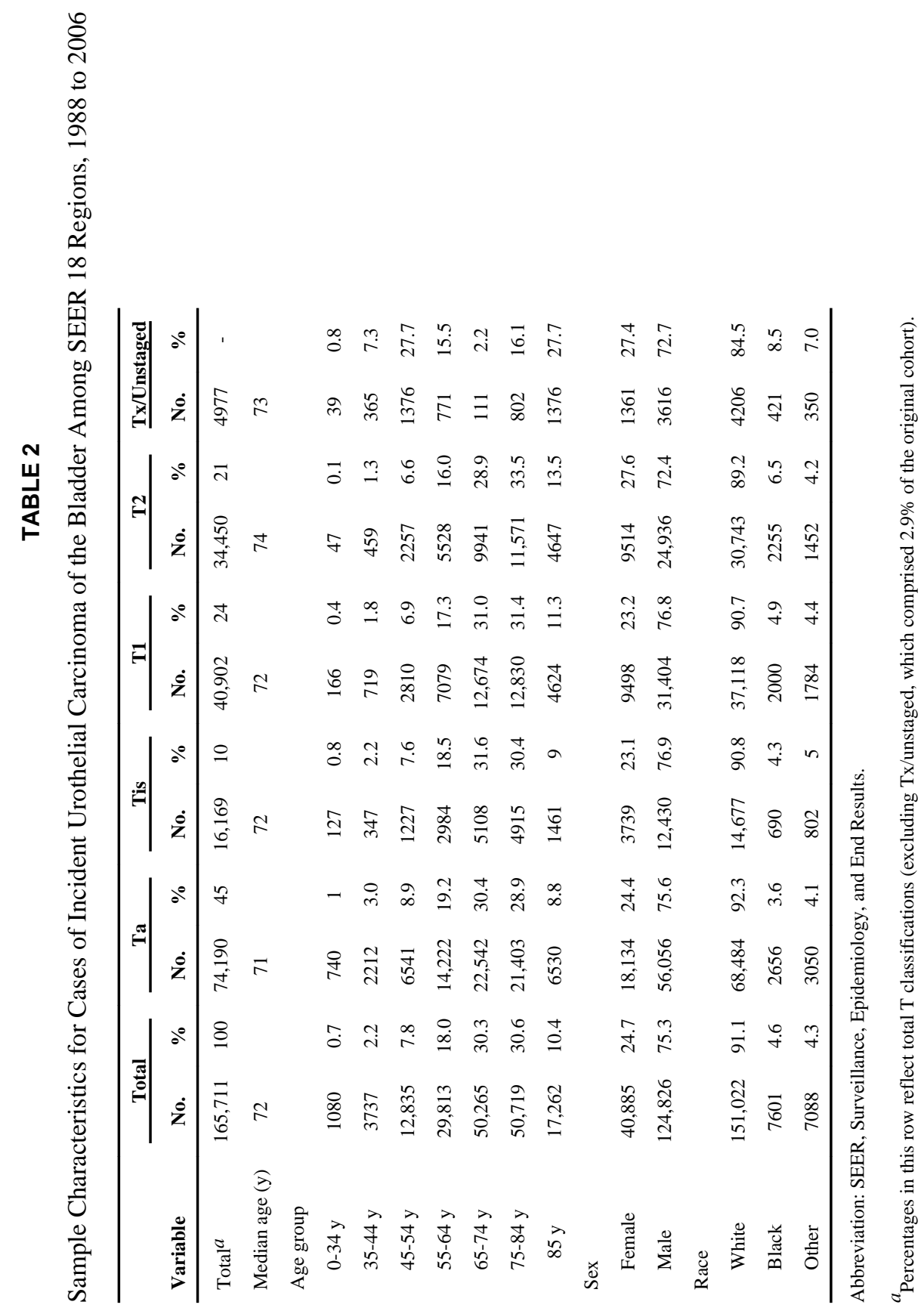


TABLE 3

Tests for Significant Changes in Adjusted Incidence Rates of Urothelial Carcinoma of the Bladder

\begin{tabular}{|c|c|c|c|c|c|c|}
\hline \multirow[b]{2}{*}{ T Classification } & \multicolumn{2}{|c|}{ Carriere and Roos Results ${ }^{14 a}$} & \multicolumn{4}{|c|}{ Joinpoint Regression Results $b$} \\
\hline & $\begin{array}{l}\text { Test Within Stage } \\
1988 \text { Versus 2006 }\end{array}$ & $P$ & $\begin{array}{c}\text { Joinpoint } \\
\text { Location, Year }\end{array}$ & $\operatorname{APC1}(95 \%$ CI $)$ & APC2 $(95 \%$ CI) & $\operatorname{AAPC}(95 \%$ CI $)$ \\
\hline $\mathrm{Ta}$ & 5.52 vs 9.09 & $<.0001$ & 1991 & $11.2(0.3-23.2)^{d}$ & $1.8(1.3-2.3)^{d}$ & $3.3(1.7-5.0)^{d}$ \\
\hline Tis & 2.57 vs 1.19 & $<.0001$ & 2003 & $-3.0(-3.7 \text { to }-2.4)^{d}$ & $-14.4(-20.5 \text { to }-7.9)^{d}$ & $-5.0(-6.2 \text { to }-3.9)^{d}$ \\
\hline $\mathrm{T} 1$ & 6.65 vs 4.61 & $<.0001$ & 1997 & $-4.6(-5.9 \text { to }-3.2)^{d}$ & $1.6(0.5-2.6)^{d}$ & $-1.6(-2.4 \text { to }-0.8)^{d}$ \\
\hline$\geq T 2$ & 3.87 vs 3.86 & .9491 & - & $0.1(-0.2$ to 0.3$)$ & - & $0.1(-0.2$ to 0.3$)$ \\
\hline Tx/unstaged & 0.73 vs 0.37 & $<.0001$ & - & $-5.1(-6.0 \text { to }-4.3)^{d}$ & & $-5.1(-6.0 \text { to }-4.3)^{d}$ \\
\hline
\end{tabular}

Abbreviations: 95\% CI, 95\% confidence interval; AAPC, average annual percentage change; APC, annual percentage change.

${ }^{a}$ Within-stage comparisons over the study period.

${ }^{b}$ APC and AAPC shown by T classification (adjusted for age, race, and sex).

${ }^{c}$ Within-stage comparisons over the study period reflect incidence rates adjusted for age, race, and sex (per 100,000) at the first (1988) and last (2006) years of the study period.

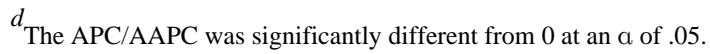


TABLE 4

Sensitivity Analyses For Significant Changes in Adjusted Incidence Rates: Within-Stage Comparisons Over the Study Period, APC and AAPC by T Classification Adjusted for Age, Race, and Sex

\begin{tabular}{|c|c|c|c|c|c|c|c|c|}
\hline & \multicolumn{4}{|c|}{ Carriere and Roos Results ${ }^{14}$} & \multicolumn{3}{|c|}{ Joinpoint Regression Results } & \multirow[b]{2}{*}{ AAPC $(95 \%$ CI $)$} \\
\hline & T Classification & $\begin{array}{c}\text { Test } \\
\text { Within } \\
\text { Stage: } \\
1988 \\
\text { Versus } \\
\text { 2006 }^{a}\end{array}$ & $P$ & $\begin{array}{c}\text { Joinpoint } \\
\text { Location, Year }\end{array}$ & APC1 $(95 \%$ CI $)$ & APC2 $(95 \%$ CI $)$ & APC3 $(95 \%$ CI $)$ & \\
\hline Reclassifying & $\mathrm{Ta}_{\text {reclassified }}$ & 7.31 vs 9.55 & $<.0001$ & 1991,2004 & $+7.4(1.3-13.9)^{b}$ & $+1.0(0.6-1.4)^{b}$ & $\begin{array}{l}-2.7(-7.8 \text { to } \\
2.7)\end{array}$ & $+1.6(0.5-2.7)^{b}$ \\
\hline low-grade & Tis $_{\text {reclassified }}$ & 0.78 vs 0.73 & .4561 & 2003 & $+0.7(0.0-1.3)^{b}$ & $\begin{array}{l}-6.2(-11.3 \text { to } \\
-0.8)^{b}\end{array}$ & & $\begin{array}{l}-0.5(-1.5 \text { to } \\
0.5)\end{array}$ \\
\hline \multicolumn{9}{|l|}{ Tis to Ta } \\
\hline & $\mathrm{Ta}$ & 5.52 vs 9.09 & $<.0001$ & 1991 & $+11.2(0.3-23.2)^{b}$ & $+1.8(1.3-2.3)^{b}$ & & $+3.3(1.7-5.0)^{b}$ \\
\hline & Tis & 2.57 vs 1.19 & $<.0001$ & 2003 & $\begin{array}{l}-3.0(-3.7 \text { to } \\
-2.4)^{b}\end{array}$ & $\begin{array}{l}-14.4(-20.5 \text { to } \\
-7.9)^{b}\end{array}$ & & $\begin{array}{l}-5.0(-6.2 \text { to } \\
-3.9)^{b}\end{array}$ \\
\hline Reciassifying & Tx/unstaged & 0.73 vs 0.37 & $<.0001$ & & $\begin{array}{l}-5.1(-6.0 \text { to } \\
-4.3)^{b}\end{array}$ & & & $\begin{array}{l}-5.1(-6.0 \text { to } \\
-4.3)^{b}\end{array}$ \\
\hline $\begin{array}{l}\text { Tx/unstaged } \\
\text { to Ta }\end{array}$ & $\begin{array}{l}\mathrm{Ta}+\mathrm{Tx} / \\
\text { unstaged }\end{array}$ & 6.25 vs 9.46 & $<.0001$ & 1991 & $+9.7(0.1-20.2)^{b}$ & $+1.3(0.9-1.8)^{b}$ & & $+2.7(1.2-4.2)^{b}$ \\
\hline
\end{tabular}

Abbreviations: $95 \%$ CI, 95\% confidence interval; AAPC, average annual percentage change; APC, annual percentage change.

${ }^{a}$ Within-Stage comparisons over the study period reflect incidence rates adjusted by age, race, and sex (per 100,000) at the first (1988) and last (2006) years of the study period.

${ }^{b}$ The APC/AAPC was significantly different from 0 at an $a$ of .05. 